Corrigendum

\title{
Corrigendum to "Closely Spaced MEG Source Localization and Functional Connectivity Analysis Using a New Prewhitening Invariance of Noise Space Algorithm"
}

\author{
Junpeng Zhang, ${ }^{1,2}$ Yuan Cui, ${ }^{3}$ Lihua Deng, ${ }^{1}$ Ling He, ${ }^{1}$ Junran Zhang, ${ }^{1}$ Jing Zhang, \\ Qun Zhou, ${ }^{1}$ Qi Liu, ${ }^{1}$ and Zhiguo Zhang ${ }^{2}$ \\ ${ }^{1}$ Department of Medical Information Engineering, School of Electrical Engineering and Information, Sichuan University, \\ Chengdu 610065, China \\ ${ }^{2}$ School of Chemical and Biomedical Engineering, School of Electrical and Electronic Engineering, \\ Nanyang Technological University, Singapore 639798 \\ ${ }^{3}$ School of Humanities and Information Management, Chengdu Medical College, Chengdu 610083, China
}

Correspondence should be addressed to Junpeng Zhang; junpeng.zhang@gmail.com

Received 22 May 2016; Accepted 1 June 2016

Copyright (C) 2016 Junpeng Zhang et al. This is an open access article distributed under the Creative Commons Attribution License, which permits unrestricted use, distribution, and reproduction in any medium, provided the original work is properly cited.

In the article titled "Closely Spaced MEG Source Localization and Functional Connectivity Analysis Using a New Prewhitening Invariance of Noise Space Algorithm" [1], there was an error in the legend of Figure 7, which should be corrected as follows.

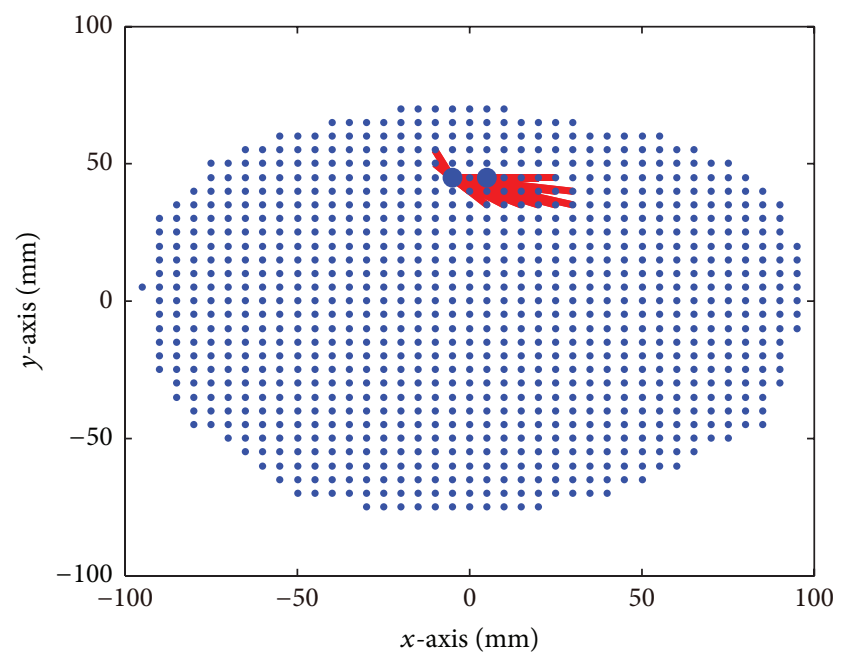

(a)

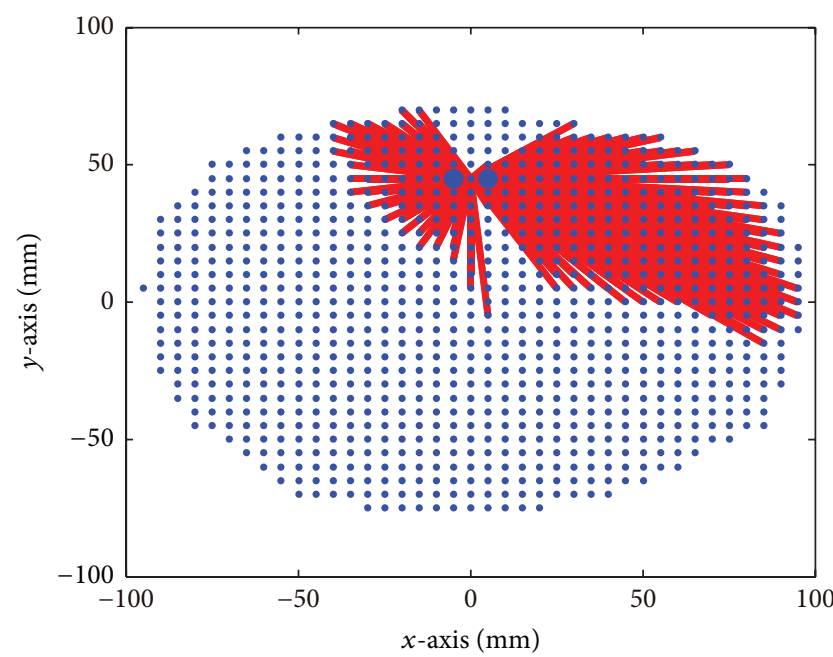

(d)

FIgure 7: Continued. 


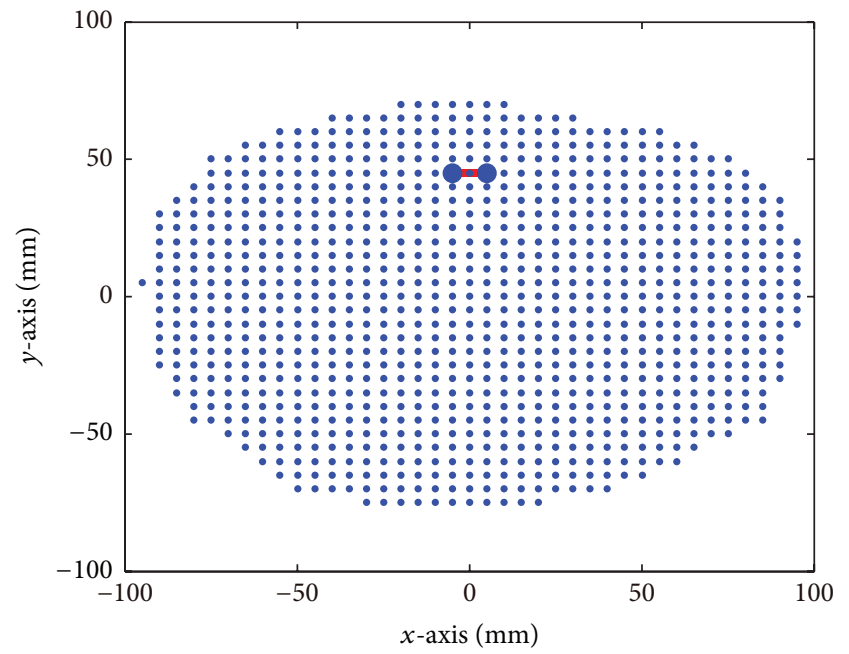

(b)

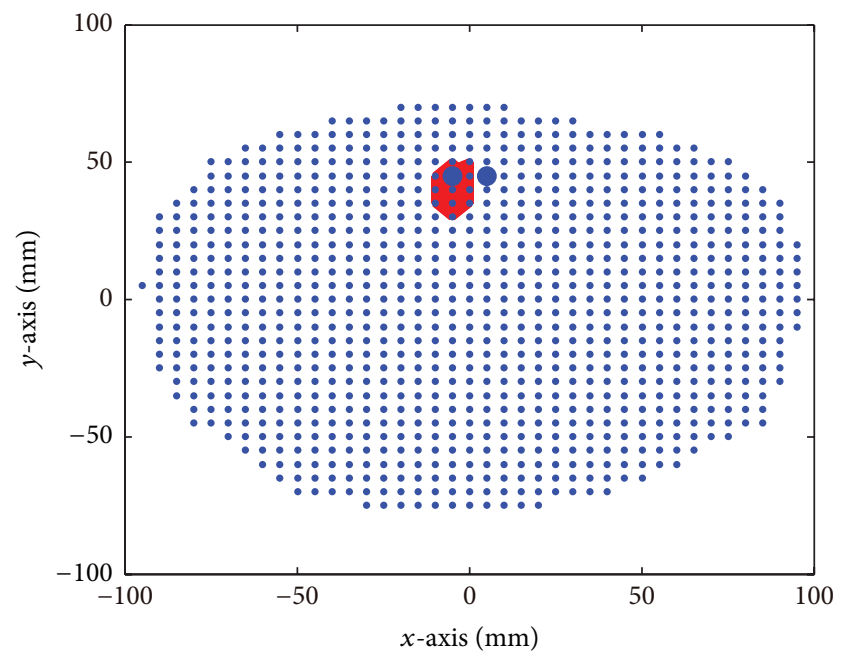

(c)

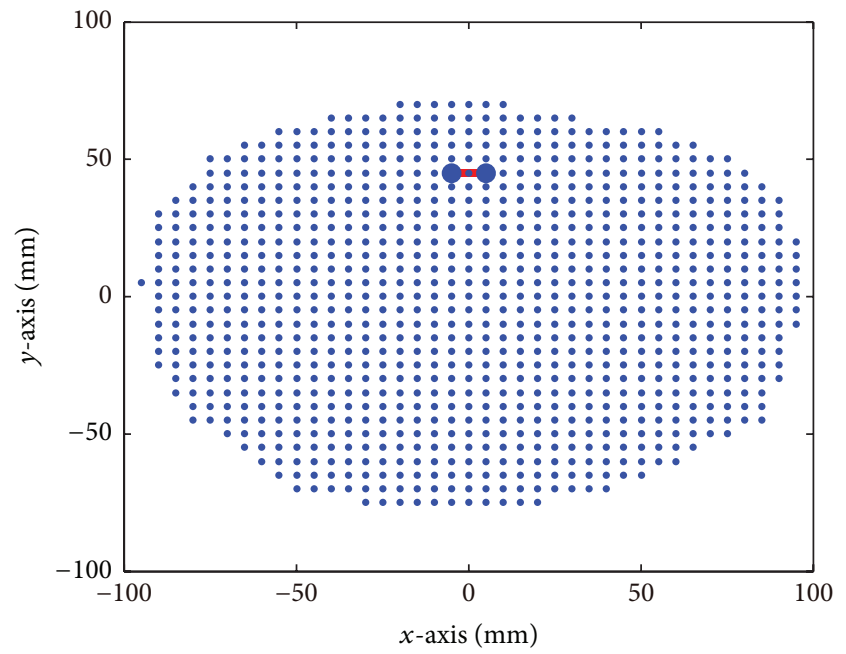

(e)

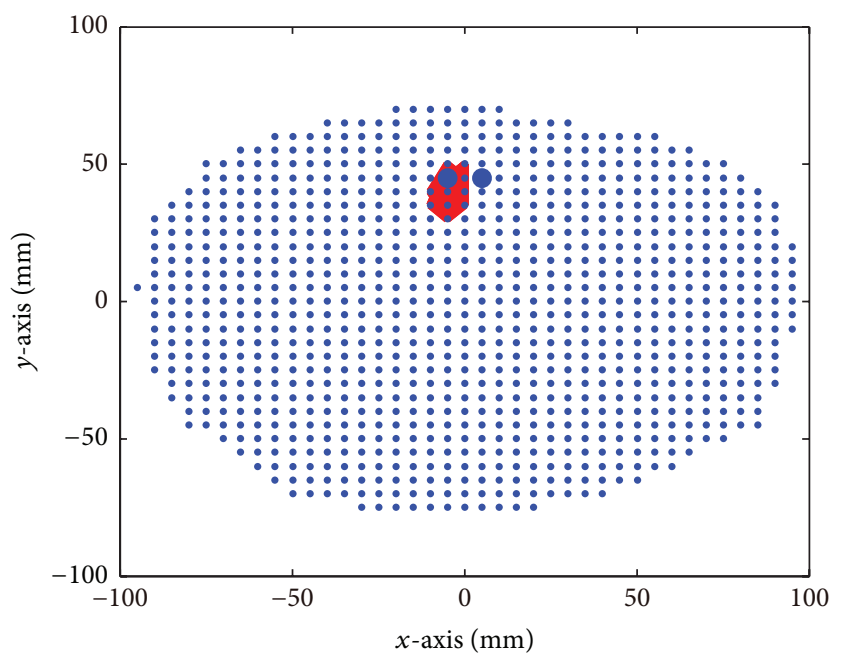

(f)

FIGURE 7: FC graphs based on source reconstruction results using LCMV beamformer, PW-INN, and sLORETA. Real MEG noise was added such that SNR = 18. Small blue dots indicate brain volume grid points, and large blue dots indicate true source locations. (a)-(c) FC graphs estimated from LCMV beamformer (a), PW-INN (b), and sLORETA (c) when $r^{2}=0$ between two sources. (d) -(f) FC graphs estimated from LCMV beamformer (d), PW-INN (e), and sLORETA (f), when $r^{2}=0.95$ between two sources.

\section{References}

[1] J. Zhang, Y. Cui, L. Deng et al., "Closely spaced MEG source localization and functional connectivity analysis using a new prewhitening invariance of noise space algorithm," Neural Plasticity, vol. 2016, Article ID 4890497, 12 pages, 2016. 

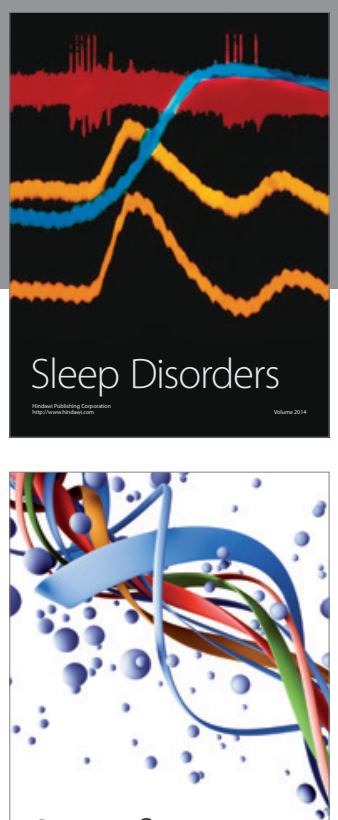

Scientifica
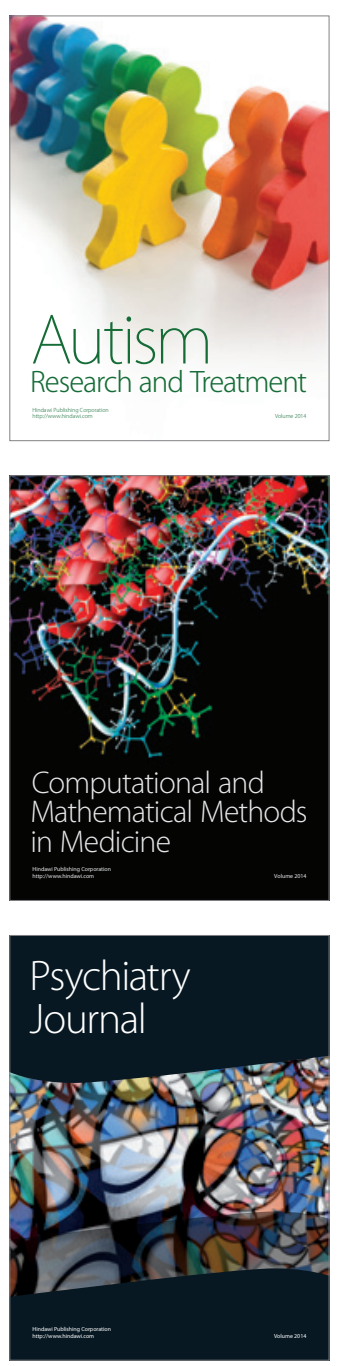
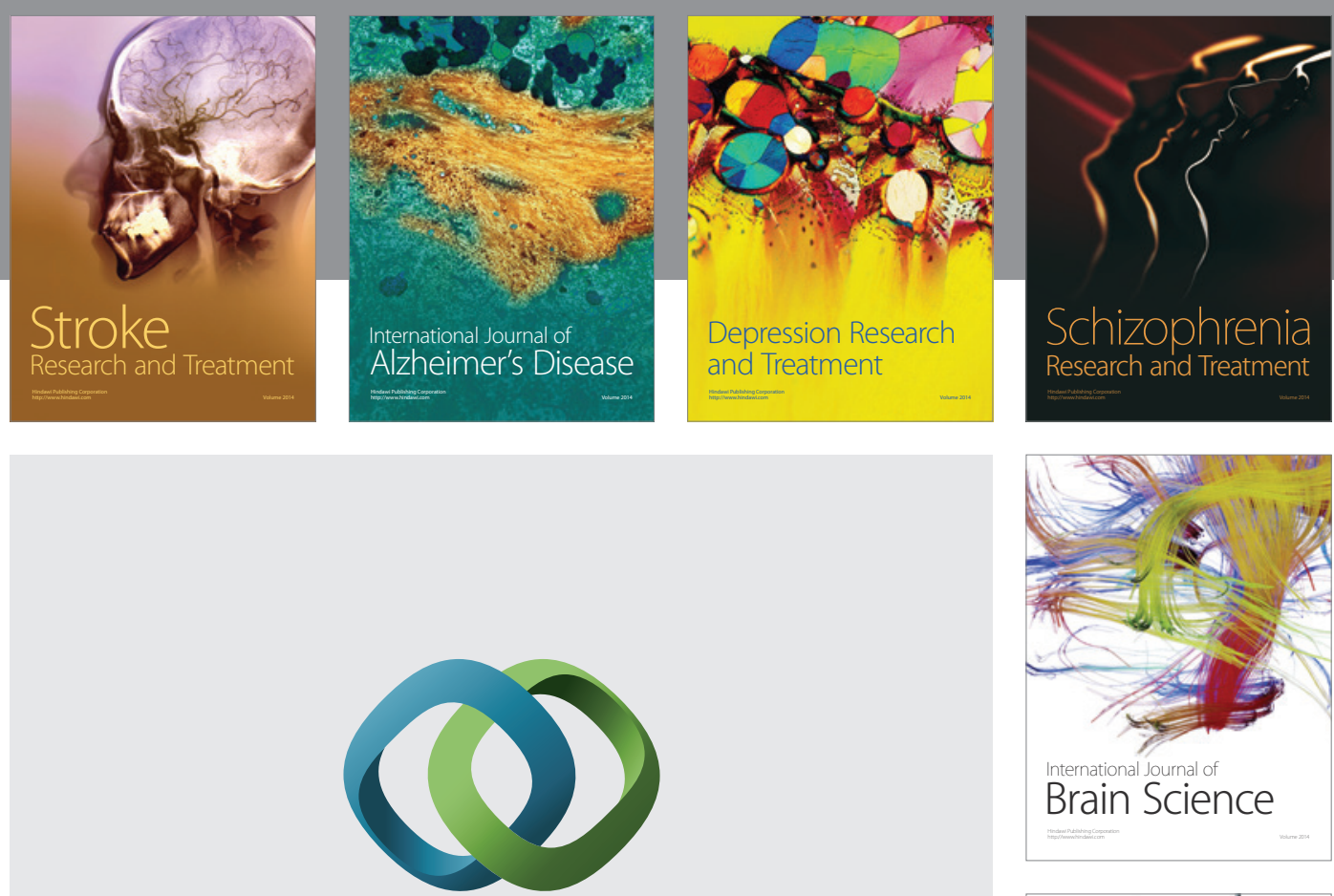

\section{Hindawi}

Submit your manuscripts at

http://www.hindawi.com
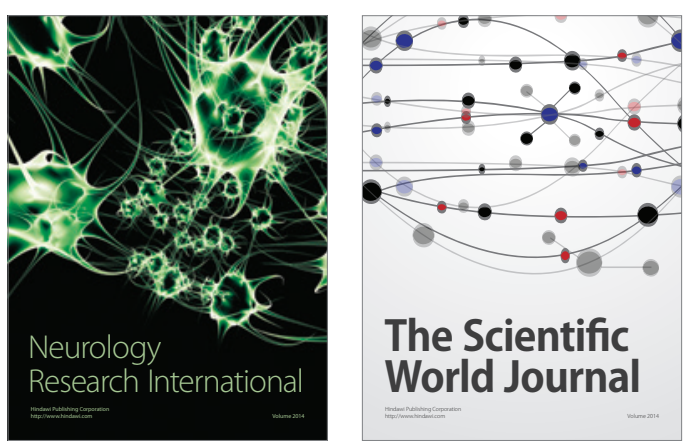

The Scientific World Journal

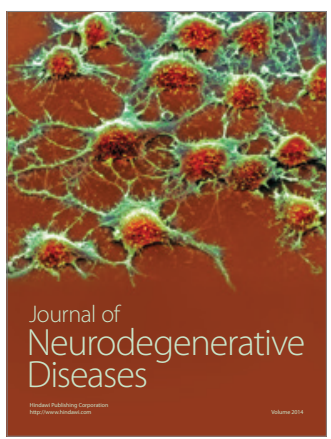

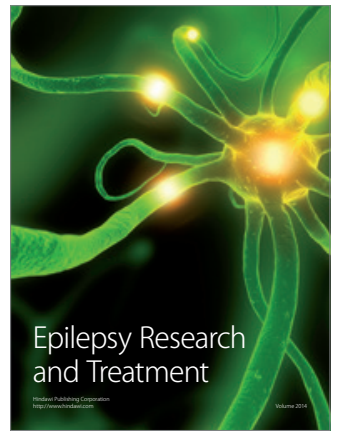

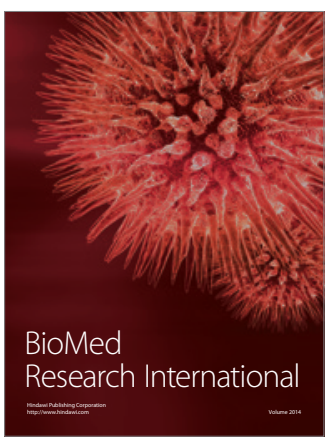

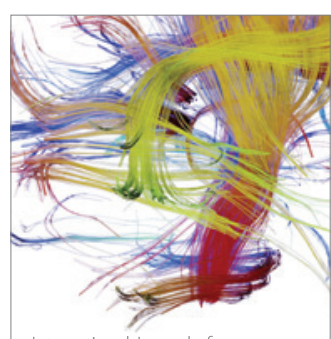

Brain Science

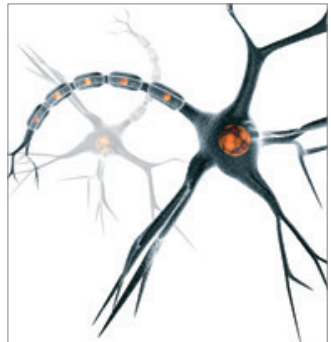

Neural Plasticity
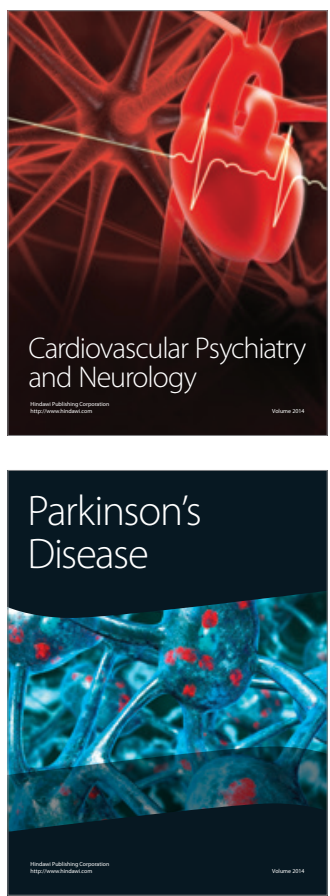